\title{
Extension of Cherenkov Light LDF Approximation for Yakutsk EAS Array
}

\author{
A. A. Al-Rubaiee, ${ }^{1,2}$ Y. Al-Douri, ${ }^{2}$ and U. Hashim ${ }^{2}$ \\ ${ }^{1}$ Department of Physics, College of Science, The University of Mustansiriyah, 10052 Baghdad, Iraq \\ ${ }^{2}$ Institute of Nano Electronic Engineering, University of Malaysia Perlis, 01000 Kangar, Malaysia
}

Correspondence should be addressed to A. A. Al-Rubaiee; dr.ahmedrubaiee@gmail.com

Received 27 May 2014; Accepted 24 July 2014; Published 14 August 2014

Academic Editor: Luciano Nicastro

Copyright (C) 2014 A. A. Al-Rubaiee et al. This is an open access article distributed under the Creative Commons Attribution License, which permits unrestricted use, distribution, and reproduction in any medium, provided the original work is properly cited.

\begin{abstract}
The simulation of the Cherenkov light lateral distribution function (LDF) in extensive air showers (EAS) was performed using CORSIKA code for configuration of Yakutsk EAS array at high energy range for different primary particles $\left(\mathrm{p}, \mathrm{Fe}\right.$, and $\left.\mathrm{O}_{2}\right)$ and different zenith angles. Depending on Breit-Wigner function a parameterization of Cherenkov light LDF was reconstructed on the basis of this simulation as a function of primary energy. A comparison of the calculated Cherenkov light LDF with that measured on the Yakutsk EAS array gives the possibility of identification of the particle initiating the shower and determination of its energy in the knee region of the cosmic ray spectrum. The extrapolation of approximated Cherenkov light LDF for high energies was obtained for primary proton and iron nuclei.
\end{abstract}

\section{Introduction}

Study of the energy spectrum and mass composition of primary cosmic rays (PCRs) in the energy range $10^{13}-10^{17} \mathrm{eV}$ is of a special interest in connection with observed index change of PCR spectrum close to $E=3 \mathrm{PeV}$ which is called the "knee" region $[1,2]$. The Cherenkov light emitted in the atmosphere by relativistic electrons of cosmic rays (CRs) in EAS carries important information about the shower development and PCR particles. The Cherenkov light LDF depends on energy and type of the primary particle, observation level, height of the first interaction, and direction of shower axis [3]. The Monte Carlo method is one of the necessary tools of numerical simulation for investigation of EAS characteristics and experimental data processing and analysis (determination of the primary particle energy type and direction of shower axis from the characteristics of Cherenkov radiation of secondary charged particles).

Agnetta et al. [4] have discussed the simulation and the experimental setup with detailed information on the detection of Cherenkov light method in EAS. On the other side, Akchurin et al. [5] have presented detailed measurements of high-energy electromagnetic and hadronic shower profiles. The Cherenkov light LDF generated in the shower development process was measured for electrons in the energy range $8-200 \mathrm{GeV}$. The Cherenkov light profiles are discussed and compared with results of Monte Carlo simulations. Berezhnev et al. [6] have installed the Cherenkov light EAS array (Tunka-133). This array permits a detailed study of cosmic ray energy spectrum and mass composition in the energy range $10^{16}-10^{18} \mathrm{eV}$ with a uniform method. The analysis of LDF and time structure of EAS Cherenkov light allowed estimating the depth of the EAS maximum $X_{\max }$.

In the present work the simulation of Cherenkov light LDF for conditions and configurations of Yakutsk EAS array $[7,8]$ is performed with the CORSIKA code $[9,10]$ using two models for simulation of hadronic processes which are QGSJET [11] and GHEISHA [12] models and EGS4 code for the simulation of the EAS electromagnetic component and Cherenkov light radiation. The approximation of the results of numerical simulation of Cherenkov light density was performed on the bases on Breit-Wigner functions [13, 14], an approach to the description of the lateral distribution of EAS Cherenkov light, and analyzes the possibility of its application for the reconstruction of the events registered on the Yakutsk array. The main advantage of this approach is 
to reconstruct the events of Cherenkov radiation measured with Yakutsk array. The comparison of the approximated Cherenkov light LDF with the reconstructed EAS events registered with Yakutsk EAS Cherenkov array has shown a good opportunity of primary particle identification and definition of its energy around the knee region.

\section{Simulation of Cherenkov Light LDF}

The simulation of Cherenkov light LDF from EAS was performed using the CORSIKA (COsmic Ray SImulations for KAscade) software package $[9,10]$ within two models: QGSJET (Quark Gluon String model with JETs) code [11] to model interactions of hadrons with energies exceeding $80 \mathrm{GeV}$ and GHEISHA (Gamma Hadron Electron Interaction SHower) code [12] for energies lower than $80 \mathrm{GeV}$. The CORSIKA code simulates the interactions and decays of various nuclei, hadrons, muons, electrons, and photons in the atmosphere. The particles are tracked through the atmosphere until they undergo reactions with an air nucleus or, in the case of unstable secondary particles, they decay [9]. The result of the simulations is detailed about the type, energy, momenta, location, and arrival time of the produced secondary particles at a given selected altitude above sea level.

The Yakutsk EAS array consists of 48 Cherenkov light detectors (500 $\mathrm{m}$ spacing between detectors); the observation level was assumed to be $100 \mathrm{~m}$ above sea level $\left(1020 \mathrm{~g} / \mathrm{cm}^{2}\right)$ and wavelength range from 300 to $600 \mathrm{~nm}$ [8].

\section{Parameterization of Cherenkov Radiation Density in EAS}

The Cherenkov LDF is a function to describe the lateral variation of Cherenkov flux with the core distance that is widely used in event reconstruction, aiming to obtain information about primary particles. Integration over the total range of core distance of LDF results in the shower size, that is, total number of particles. Estimating of core position and age parameter is also made by using the total number of Cherenkov photons $\left(N_{\gamma}\right)$ radiated by electrons in EAS which is directly proportional to the primary energy $E_{0}[15]$ :

$$
N_{\gamma}\left(E_{0}\right) \approx 3.7 \times 10^{3} \frac{E_{0}}{\xi_{e}} \approx 4.5 \times 10^{10} \frac{E_{0}}{10^{15} \mathrm{eV}},
$$

where $\xi_{e}$ is the critical energy of electrons which equals 81.4 MeV. The experimental measurement of this magnitude is rather difficult, so one can use the density of Cherenkov radiation, the number of photons $\left(\Delta N_{\gamma}\right)$ per unit detector area $(\Delta S)$, which appears as a function of energy and distance from the shower axis [16]:

$$
Q\left(E_{0}, R\right)=\frac{\Delta N_{\gamma}\left(E_{0}, R\right)}{\Delta S},
$$

where $R$ is the distance from the shower axis.

Direct measurements of Cherenkov light showed that the fluctuation of LDF in EAS is essentially less than the total number of photons $N_{\gamma}$ [1]. For parameterization of simulated
Cherenkov light LDF, we used the proposed function as a function of distance $R$ from shower axis, depth of shower maximum, and energy $E_{0}$ of the initial primary particle, which depends on four parameters $a, b, s$, and $r_{o}$ :

$$
Q\left(E_{0}, R\right)=\frac{C s \exp [a-A]}{b\left[(R / b)^{2}+\left(R-r_{o}\right)^{2} / b^{2}+R s^{2} / b\right]},
$$

where $A$ is defined as

$$
A=\frac{R}{b}+\frac{\left(R-r_{o}\right)}{b}+\left(\frac{R}{b}\right)^{2}+\frac{\left(R-r_{o}\right)^{2}}{b^{2}},
$$

where $C$ is the normalization constant [3] and $a, b, s$, and $r_{o}$ are parameters of Cherenkov light LDF. The estimation of Cherenkov light density was performed in the energy range $10^{13}-10^{17} \mathrm{eV}$ for different primary particles and different zenith angles. The energy dependence of LDF parameters is approximated as

$$
K\left(E_{0}\right)=c_{0}+c_{1} \lg \left(E_{0}\right)+c_{2}\left(\lg E_{0}\right)^{2}+c_{3}\left(\lg E_{0}\right)^{3},
$$

where $c_{0}, c_{1}, c_{2}$, and $c_{3}$ are coefficients that depend on the type of primary particles and the zenith angle (see Tables 1 and 2).

The obtained Cherenkov light LDF in EAS due to various cosmic ray particles ( $\mathrm{p}$ and $\mathrm{Fe}$ ) below and in the region of the "knee" are presented in Figure 1. It demonstrates the results of the simulated (solid lines) and parameterized (dashed lines) Cherenkov light LDF for vertical showers for primary proton and iron nuclei, respectively, at different primary energies.

The accuracy of the Cherenkov light LDF approximation with that simulated for primary proton is better than $18 \%$ at the distances 10-150 $\mathrm{m}$ from the shower axis and about 5-15\% for the other distances. The accuracy of iron nuclei was found close to $15 \%$ at the distances $10-150 \mathrm{~m}$ from the core shower and about $5-10 \%$ at the other distances.

\section{Comparison of the Approximated LDF with Yakutsk Measurements}

The Yakutsk EAS array studies cosmic rays of extremely high energies, that is, in the field of cosmic ray astrophysics, an active area at the cutting edge of basic research. The construction of Yakutsk array depends on two main goals; the first is the investigation of cascades of elementary particles in atmosphere initiated by primary particles and the other is the reconstruction of astrophysical properties of the primaries: intensity, energy spectrum, mass composition, and their origin [8]. The main parameters of EAS measurements are zenith and azimuth angles, shower core location, individual LDF, and the density of Cherenkov radiation $Q(R)$. The possibility for reconstruction of the type of EAS primary particles can be demonstrated in Figures 2 and 3.

Figure 2(a) demonstrates the comparison of approximated Cherenkov light LDF (dash lines) with that measured with Yakutsk EAS array (symbols) for three primaries $\left(\mathrm{p}, \mathrm{O}_{2}\right.$, and $\mathrm{Fe}$ ) at the distance 100 to $400 \mathrm{~m}$ from the shower core.

In Figure 2(b), one may see reasonable agreement between the approximated Cherenkov light LDF (dash lines) and 
TABLE 1: Coefficients $c_{i}$ that determine the energy dependence (5) of the extrapolated parameters $a, b, s$, and $r_{o}$ for primary proton, iron nuclei, and primary oxygen for vertical showers of Yakutsk Cherenkov EAS array.

\begin{tabular}{|c|c|c|c|c|}
\hline & $c_{0}$ & $\theta=$ & $c_{2}$ & $c_{3}$ \\
\hline \multicolumn{5}{|c|}{$\mathrm{P}$} \\
\hline$a$ & $1.026 \cdot 10^{2}$ & $-2.615 \cdot 10^{1}$ & $2.063 \cdot 10^{0}$ & $-4.914 \cdot 10^{-2}$ \\
\hline$b$ & $-9.709 \cdot 10^{0}$ & $2.117 \cdot 10^{0}$ & $-1.439 \cdot 10^{-1}$ & $-3.190 \cdot 10^{-3}$ \\
\hline$\sigma$ & $8.126 \cdot 10^{1}$ & $-1.669 \cdot 10^{1}$ & $1.132 \cdot 10^{0}$ & $-2.548 \cdot 10^{-2}$ \\
\hline$r_{o}$ & $-1.427 \cdot 10^{1}$ & $2.918 \cdot 10^{0}$ & $-2.007 \cdot 10^{-1}$ & $4.430 \cdot 10^{-3}$ \\
\hline \multicolumn{5}{|c|}{$\mathrm{Fe}$} \\
\hline$a$ & $-3.851 \cdot 10^{2}$ & $7.187 \cdot 10^{1}$ & $-4.504 \cdot 10^{0}$ & $9.752 \cdot 10^{-2}$ \\
\hline$b$ & $-4.415 \cdot 10^{1}$ & $9.802 \cdot 10^{0}$ & $-7.120 \cdot 10^{-1}$ & $1.711 \cdot 10^{-2}$ \\
\hline$\sigma$ & $9.887 \cdot 10^{1}$ & $-2.102 \cdot 10^{1}$ & $1.481 \cdot 10^{0}$ & $-3.474 \cdot 10^{-2}$ \\
\hline$r_{o}$ & $-1.216 \cdot 10^{2}$ & $2.505 \cdot 10^{1}$ & $-1.714 \cdot 10^{0}$ & $3.877 \cdot 10^{-2}$ \\
\hline \multicolumn{5}{|c|}{$\mathrm{O}_{2}$} \\
\hline$a$ & $-9.194 \cdot 10^{1}$ & $1.328 \cdot 10^{1}$ & $-6.077 \cdot 10^{-1}$ & $1.121 \cdot 10^{-2}$ \\
\hline$b$ & $3.847 \cdot 10^{1}$ & $-7.855 \cdot 10^{0}$ & $5.431 \cdot 10^{-1}$ & $-1.254 \cdot 10^{-2}$ \\
\hline$\sigma$ & $-1.496 \cdot 10^{1}$ & $2.781 \cdot 10^{0}$ & $-1.784 \cdot 10^{-1}$ & $3.830 \cdot 10^{-3}$ \\
\hline$r_{o}$ & $-2.097 \cdot 10^{2}$ & $4.359 \cdot 10^{1}$ & $-3.014 \cdot 10^{0}$ & $6.910 \cdot 10^{-2}$ \\
\hline
\end{tabular}

TABLE 2: Coefficients $c_{i}$ that determine the energy dependence (5) of the extrapolated parameters $a, b, s$, and $r_{o}$ for primary proton and iron nuclei for inclined showers of Yakutsk Cherenkov EAS array.

\begin{tabular}{|c|c|c|c|c|}
\hline$K$ & $c_{0}$ & $c_{1}$ & $c_{2}$ & $c_{3}$ \\
\hline \multicolumn{5}{|c|}{$\theta=30 \circ$} \\
\hline \multicolumn{5}{|c|}{$\mathrm{P}$} \\
\hline$a$ & $-7.715 \cdot 10^{1}$ & $1.194 \cdot 10^{1}$ & $-6.242 \cdot 10^{-1}$ & $1.389 \cdot 10^{-2}$ \\
\hline$b$ & $-4.417 \cdot 10^{1}$ & $9.292 \cdot 10^{0}$ & $-6.400 \cdot 10^{-1}$ & $1.458 \cdot 10^{-2}$ \\
\hline$\sigma$ & $6.133 \cdot 10^{0}$ & $-1.353 \cdot 10^{0}$ & $9.383 \cdot 10^{-2}$ & $-2.180 \cdot 10^{-3}$ \\
\hline$r_{o}$ & $1.415 \cdot 10^{1}$ & $-3.037 \cdot 10^{0}$ & $2.137 \cdot 10^{-1}$ & $-5.160 \cdot 10^{-3}$ \\
\hline \multicolumn{5}{|c|}{$\theta=10^{\circ}$} \\
\hline \multicolumn{5}{|c|}{$\mathrm{P}$} \\
\hline$a$ & $1.33 \cdot 10^{2}$ & $-3.146 \cdot 10^{1}$ & $2.365 \cdot 10^{0}$ & $-5.467 \cdot 10^{-2}$ \\
\hline$b$ & $-8.468 \cdot 10^{1}$ & $1.791 \cdot 10^{1}$ & $-1.250 \cdot 10^{0}$ & $2.898 \cdot 10^{-2}$ \\
\hline$\sigma$ & $8.912 \cdot 10^{1}$ & $-1.810 \cdot 10^{1}$ & $1.218 \cdot 10^{0}$ & $-2.733 \cdot 10^{-2}$ \\
\hline$r_{o}$ & $4.368 \cdot 10^{2}$ & $-8.884 \cdot 10^{1}$ & $6.011 \cdot 10^{0}$ & $-1.356 \cdot 10^{-1}$ \\
\hline \multicolumn{5}{|c|}{$\theta=10^{\circ}$} \\
\hline \multicolumn{5}{|c|}{$\mathrm{Fe}$} \\
\hline$a$ & $-4.151 \cdot 10^{2}$ & $8.002 \cdot 10^{1}$ & $-5.195 \cdot 10^{0}$ & $1.161 \cdot 10^{-1}$ \\
\hline$b$ & $3.609 \cdot 10^{1}$ & $-7.492 \cdot 10^{0}$ & $5.262 \cdot 10^{-1}$ & $-1.232 \cdot 10^{-2}$ \\
\hline$\sigma$ & $5.043 \cdot 10^{1}$ & $-1.043 \cdot 10^{1}$ & $7.098 \cdot 10^{-1}$ & $-1.604 \cdot 10^{-2}$ \\
\hline$r_{o}$ & $-4.926 \cdot 10^{1}$ & $1.067 \cdot 10^{1}$ & $-7.673 \cdot 10^{-1}$ & $1.809 \cdot 10^{-2}$ \\
\hline
\end{tabular}

measurements in Yakutsk array (symbols) for primary proton and iron nuclei at the primary energy $5 \mathrm{PeV}$ and zenith angle $\theta=10^{\circ}$.

The extrapolation of Cherenkov light LDF parameterization for higher energies $\left(>10^{16} \mathrm{eV}\right)$ can be seen in Figure 3, where Figure 3(a) displays the comparison of the approximated Cherenkov light LDF that extrapolated to $20 \mathrm{PeV}$ (dash lines) and that LDF measured with Yakutsk EAS array (symbols) for iron nuclei at two zenith angles $\theta=0^{\circ}$ and $30^{\circ}$. To illustrate more vividly the errors in approximation (3), the function $Q$ in Figure 3(a) was multiplied by 0.8 for $\theta=10^{\circ}$.
Figure 3(b) shows the comparison of the approximated Cherenkov light LDF that extrapolated to 50 and $100 \mathrm{PeV}$ (dash lines) and LDF measured with Yakutsk EAS array (symbols) for primary proton at vertical showers. The good agreement between the model parameters of extrapolated Cherenkov light LDF as a function of primary energy of different primaries with that measured with Yakutsk array shows that this model is adequate and is usable for different Cherenkov arrays.

The parameterized Cherenkov light LDF in Figures 2 and 3 slightly differs from the LDF measured with the Yakutsk 


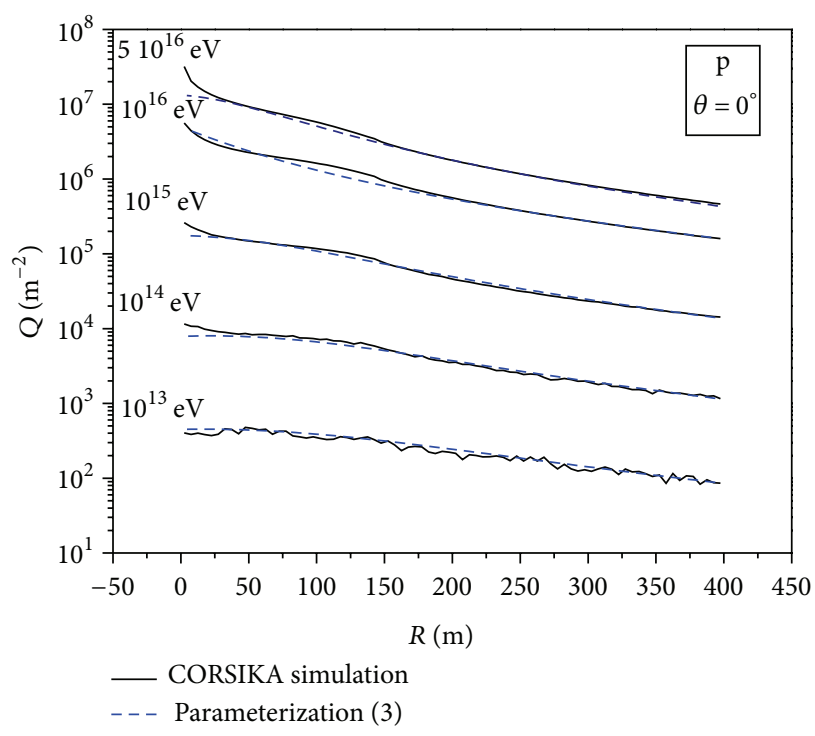

(a)

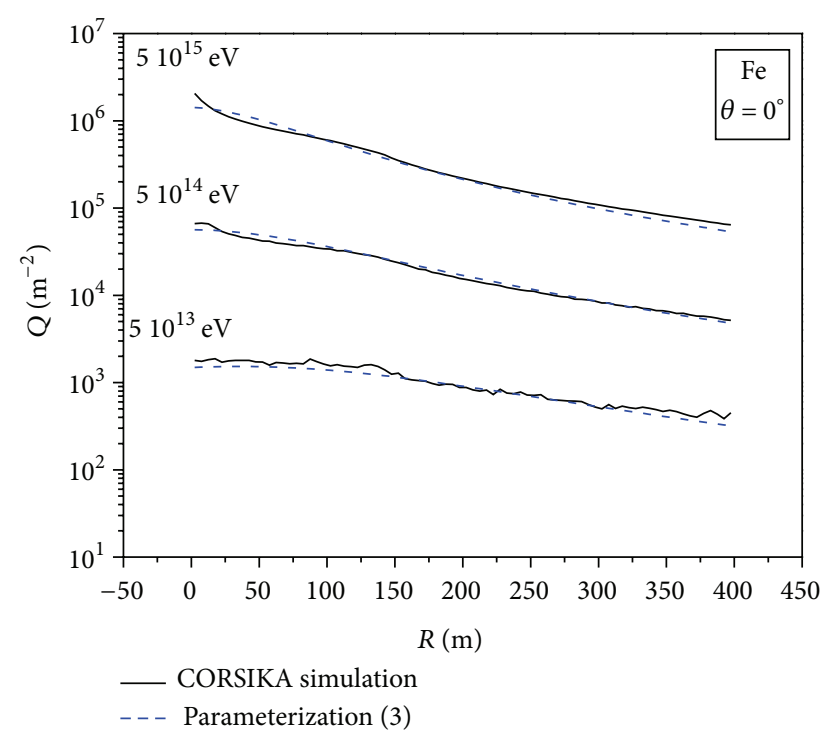

(b)

FIGURE 1: Lateral distributions of Cherenkov light that was simulated with CORSIKA code (solid lines) and one approximated (3) (dashed lines) for vertical showers initiated by (a) primary proton at the energy range $10^{13}-5 \cdot 10^{16} \mathrm{eV}$ and (b) iron nuclei at the energies $5 \cdot 10^{13}, 5 \cdot 10^{14}$, and $5 \cdot 10^{15} \mathrm{eV}$.

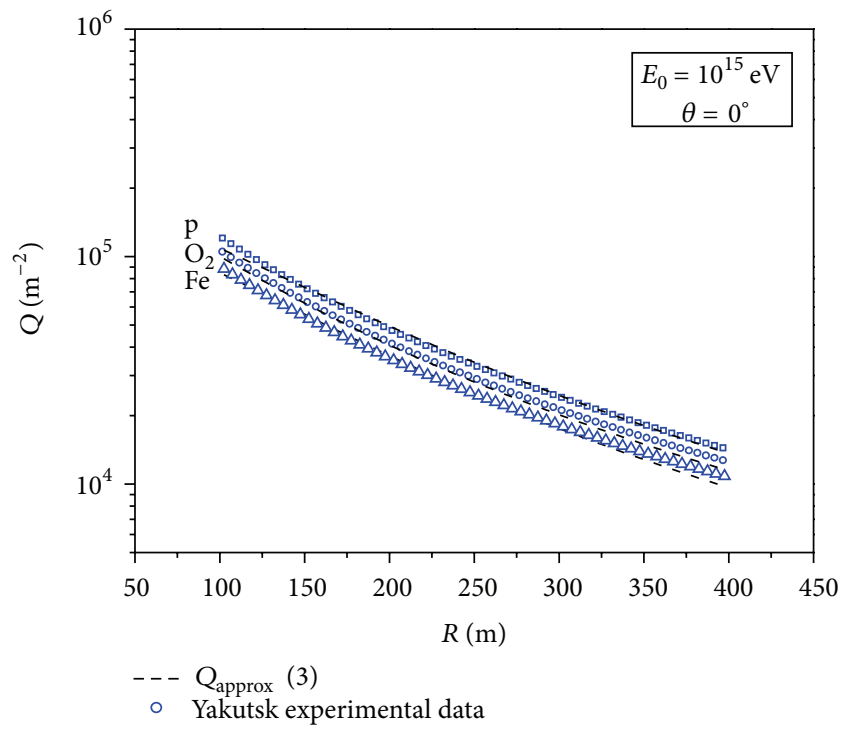

(a)

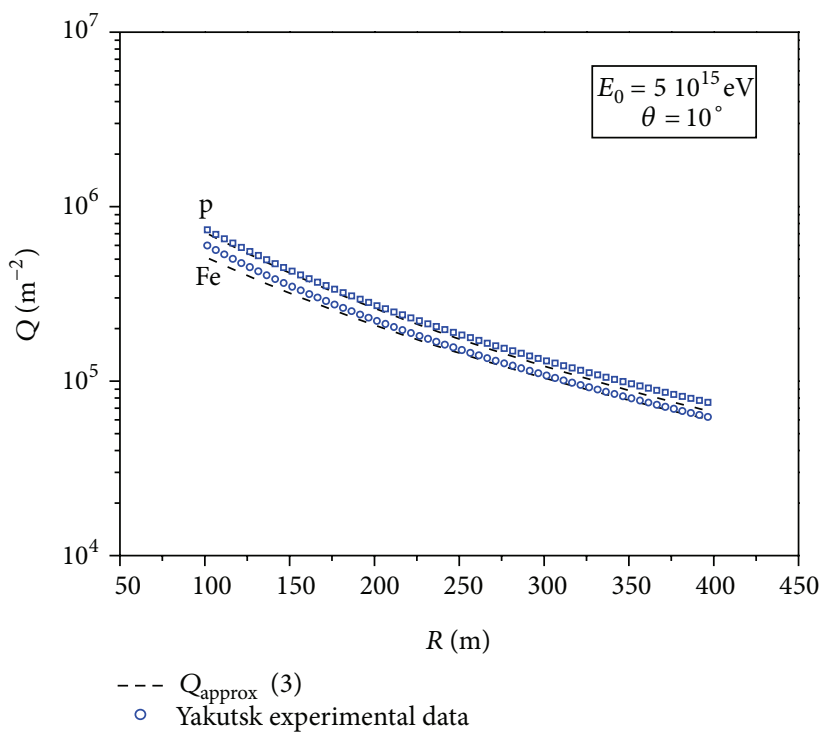

(b)

Figure 2: Comparison of the parameterized Cherenkov light LDF with the experimental data obtained by Yakutsk EAS array (symbols) for the following: (a) three different primary particles $\left(\mathrm{Fe}, \mathrm{O}_{2}\right.$, and $\mathrm{p}$ ) at the energy $10^{15}$ for vertical showers and (b) two different primary particles (Fe and $\mathrm{p}$ ) at fixed primary energy $5 \cdot 10^{15} \mathrm{eV}$ and fixed zenith angle $\left(\theta=10^{\circ}\right)$.

EAS array; at the distance interval 100-400 $\mathrm{m}$, the distinction is about $5-20 \%$ for primary proton, $3-11 \%$ for primary oxygen, and $5-13 \%$ for iron nuclei for vertical showers. For inclined showers, the distinction at the same distance interval is about $15-20 \%$ at $\theta=30^{\circ}$ for primary proton and about $8-$ $20 \%$ at $\theta=10^{\circ}$ for primary proton and iron nuclei.

\section{Conclusion}

The lateral distribution function of Cherenkov radiation from particles of extensive air showers initiated by primary proton, iron nuclei, and oxygen has been simulated in the energy range $10^{13}-10^{17} \mathrm{eV}$ using CORSIKA code. On the 


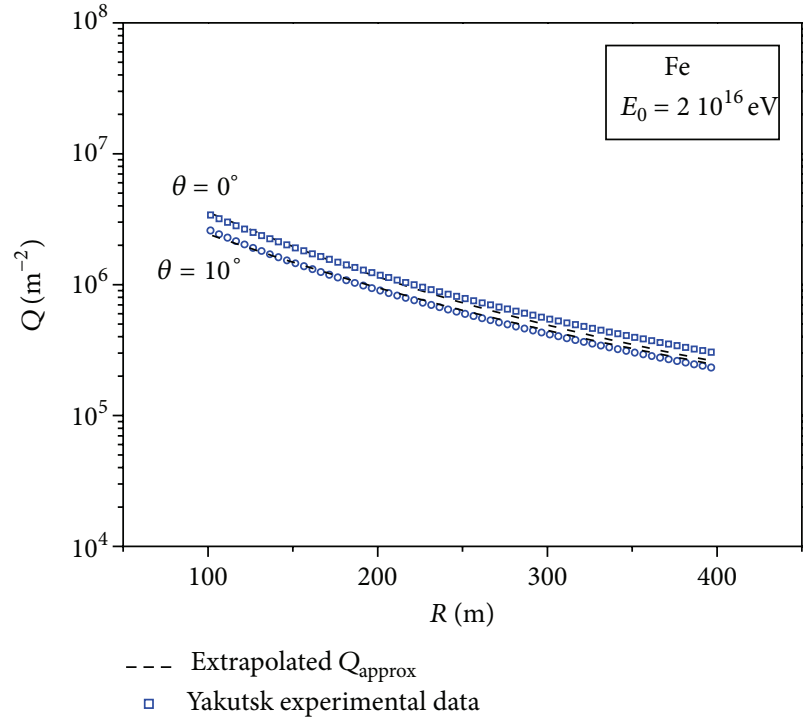

(a)

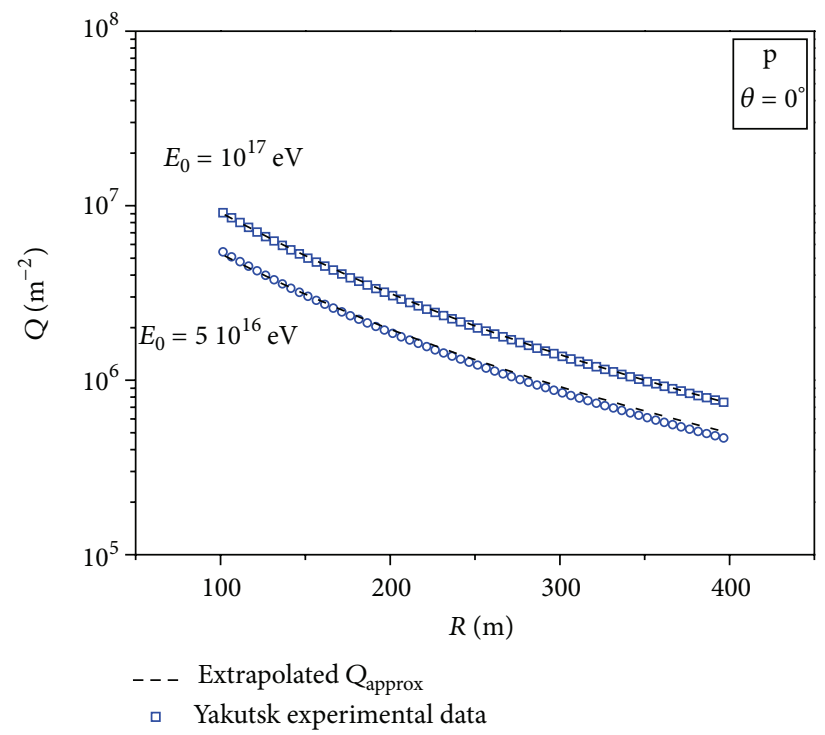

(b)

Figure 3: Extrapolation of parameterized Cherenkov light LDF (3) (dash lines) in comparison with experimental data obtained by Yakutsk EAS array (symbols) for the following: (a) iron nuclei at the energy $2 \cdot 10^{16} \mathrm{eV}$ for different zenith angles $\left(\theta=0^{\circ}\right.$ and $\left.10^{\circ}\right)$; (b) vertical showers initiated primary proton at two different energies $5 \cdot 10^{16}$ and $10^{17} \mathrm{eV}$.

basis of this simulation with depending on Breit-Wigner function; sets of approximating functions were constructed for different primary particles and different zenith angles. The comparison of the approximated Cherenkov light lateral distribution functions with that measured with Yakutsk EAS array has demonstrated the ability of identification of the particle initiating EAS showers and determination of its energy around and above the knee region of the cosmic ray spectrum. The extrapolation of the Cherenkov light lateral distribution function parameterization of the obtained data with CORSIKA program for the energies $\left(E_{0}>10^{16} \mathrm{eV}\right)$ is obtained. The main advantage of the given approach is the opportunity of creation of a representative library of lateral distribution function patterns for a short time which could be utilized for analysis of real events detected with EAS arrays and reconstruction of primary cosmic ray energy spectrum and mass composition.

\section{Conflict of Interests}

The authors declare that they have no conflict of interests regarding the publication of this paper.

\section{Acknowledgments}

Y. Al-Douri would like to acknowledge the University of Malaysia Perlis for Grant no. 9007-00111 and TWAS-Italy for the full support of his visit to JUST-Jordan under TWASUNESCO Associateship.

\section{References}

[1] G. B. Khristiansen, Y. A. Fomin, N. N. Kalmykov et al., "The primary cosmic ray mass composition around the knee of the energy spectrum," Nuclear Physics B: Proceedings Supplements, vol. 39, no. 1, pp. 235-241, 1995.

[2] M. Amenomori, Z. Cao, B. Z. Dai et al., "The cosmic-ray energy spectrum between $10^{14.5}$ and $10^{16.3} \mathrm{eV}$ covering the knee region," The Astrophysical Journa, vol. 461, pp. 453-460, 2008.

[3] A. A. Al-Rubaiee, O. A. Gress, K. S. Lokhtin, Y. V. Parfenov, and S. I. Sinegovskii, "Modeling and parameterization of the spatial distribution of Čerenkov light from extensive air showers," Russian Physics Journal, vol. 48, no. 10, pp. 1004-1011, 2005.

[4] G. Agnetta, P. Assis, B. Biondo et al., "Extensive air showers and diffused Cherenkov light detection: The ULTRA experiment," Nuclear Instruments and Methods in Physics Research Section A: Accelerators, Spectrometers, Detectors and Associated Equipment, vol. 570, pp. 22-35, 2007.

[5] N. Akchurin, K. Carrell, J. Hauptman et al., "Comparison of high-energy electromagnetic shower profiles measured with scintillation and Cherenkov light," Nuclear Instruments and Methods in Physics Research A: Accelerators, Spectrometers, Detectors and Associated Equipment, vol. 548, no. 3, pp. 336354, 2005.

[6] S. F. Berezhnev, D. Besson, N. M. Budnev et al., "The Tunka-133 EAS Cherenkov light array: status of 2011," Nuclear Instruments and Methods in Physics Research A: Accelerators, Spectrometers, Detectors and Associated Equipment, vol. 692, pp. 98-105, 2012.

[7] S. Knurenko, V. Kolosov, Z. Petrov, I. Sleptsov, and S. Starostin, in Proceedings of the 28th International Cosmic Ray Conference (ICRC '03), pp. 177-179, Tsukuba, Japan, 2003.

[8] A. A. Ivanov, S. P. Knurenko, and I. Y. Sleptsov, "Measuring extensive air showers with Cherenkov light detectors of the Yakutsk array: the energy spectrum of cosmic rays," New Journal of Physics, vol. 11, Article ID 065008, 30 pages, 2009.

[9] D. Heck and T. Peirog, Extensive Air Shower Simulations at the Highest Energies-A User's Guide, Institut fur Kernphysik, Heidelberg, Germany, 2013. 
[10] J. Knapp, D. Heck, S. J. Sciutto, M. T. Dova, and M. Risse, "Extensive air shower simulations at the highest energies," Astroparticle Physics, vol. 19, no. 1, pp. 77-99, 2003.

[11] S. Ostapchenko, "QGSJET-II: towards reliable description of very high energy hadronic interactions," Nuclear Physics B: Proceedings Supplements, vol. 151, pp. 143-146, 2006.

[12] D. Heck and R. Engel, in Proceedings of the 28th International Cosmic Ray Conference, pp. 279-282, Tsukuba, Japan, 2003.

[13] S. C. Mavrodiev, A. L. Mishev, and J. N. Stamenov, "A method for energy estimation and mass composition determination of primary cosmic rays at the Chacaltaya observation level based on the atmospheric Cherenkov light technique," Nuclear Instruments and Methods in Physics Research A: Accelerators, Spectrometers, Detectors and Associated Equipment, vol. 530, no. 3, pp. 359-366, 2004.

[14] A. Mishev, "Analysis of lateral distribution of atmospheric cherenkov light at high mountain altitude towards event reconstruction," ISRN High Energy Physics, vol. 2012, Article ID 906358, 12 pages, 2012.

[15] N. Aliev, T. Alimov, M. Kakhkharov et al., in Proceedings of the 18th International Cosmic Ray Conference (ICRC '83), vol. 2, pp. 383-386, Bangalore, India, 1983.

[16] A. Mishev, I. Angelov, E. Duverger, R. Gschwind, L. Makovicka, and J. Stamenov, "Experimental study and Monte Carlo modeling of the Cherenkov effect," Nuclear Instruments and Methods in Physics Research A: Accelerators, Spectrometers, Detectors and Associated Equipment, vol. 474, no. 2, pp. 101-107, 2001. 

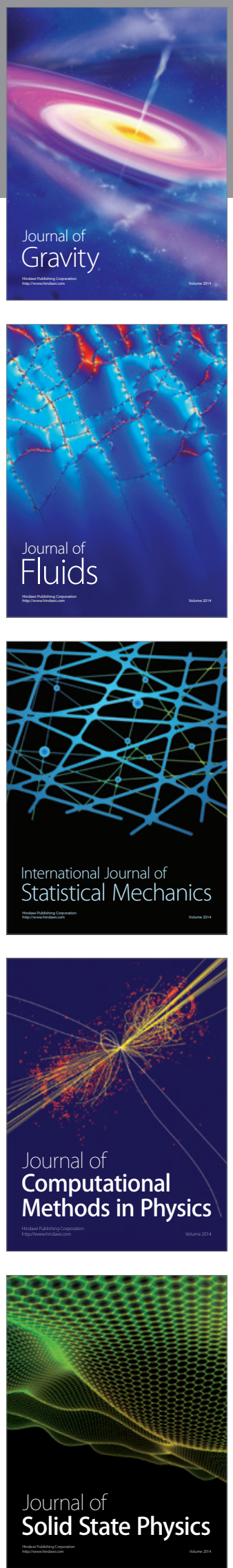

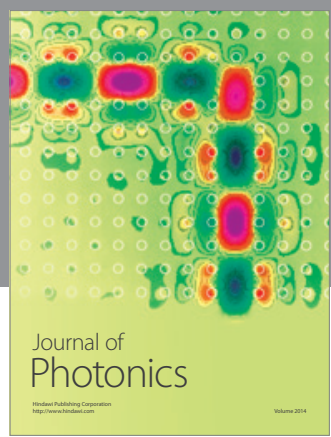

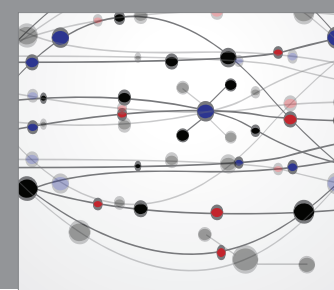

The Scientific World Journal

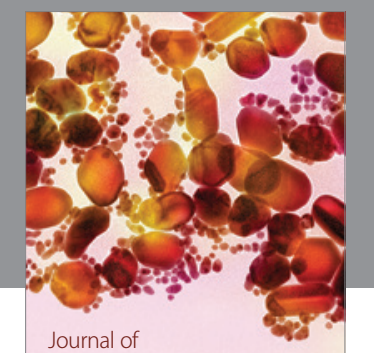

Soft Matter
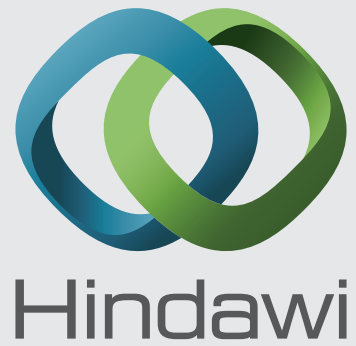

Submit your manuscripts at

http://www.hindawi.com
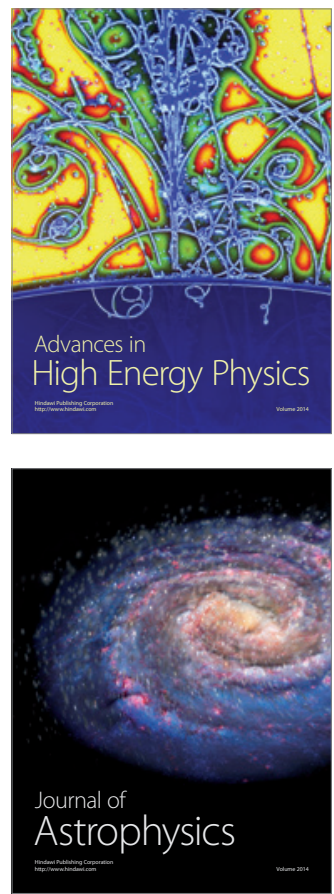
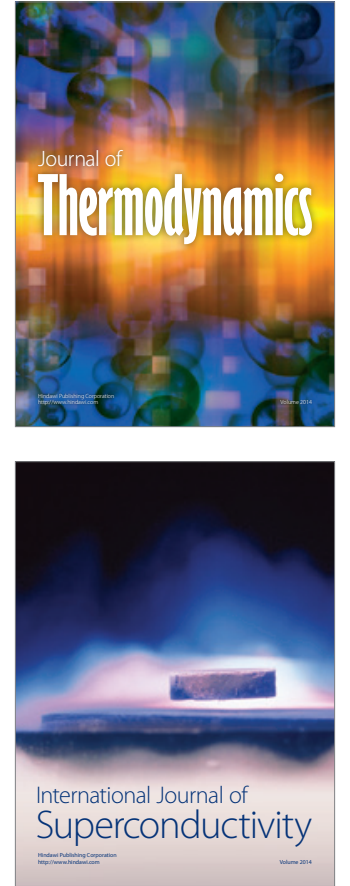
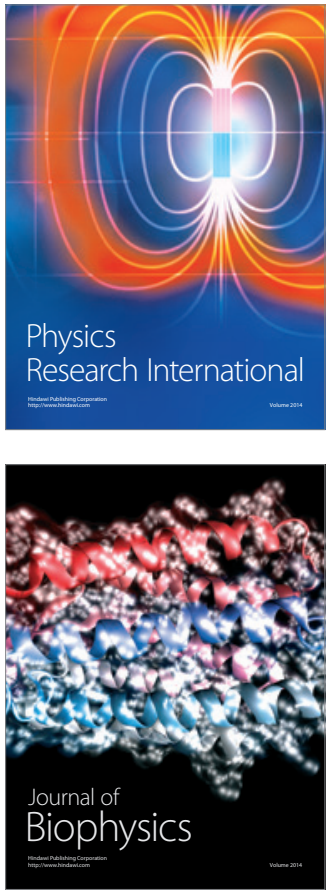
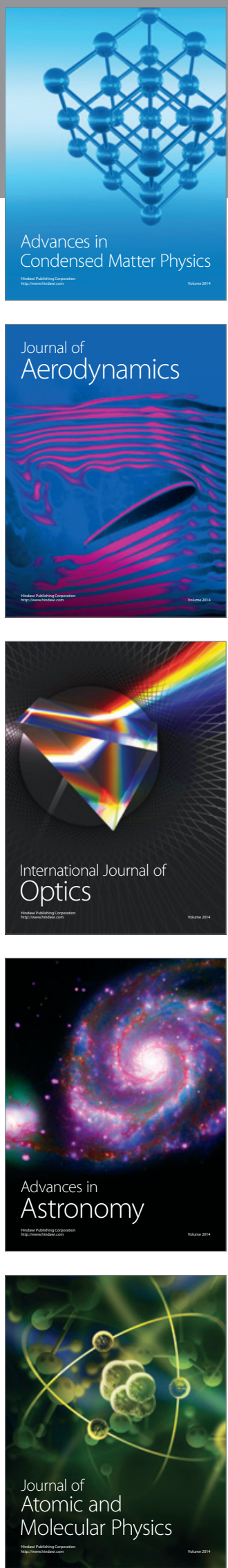